\title{
A NOTE ON THE RIEMANN ZETA FUNCTION
}

\section{RAYMOND AYOUB}

The object of this note is to add one more formula to the vast literature on the Riemann zeta function. ${ }^{1}$ The formula does not appear to have any immediate application but is interesting in that it is a kind of interpolation formula. ${ }^{2}$ Specifically we shall prove

ThEOREM. If $s$ is not an integer $s=\sigma+i t$ then

$$
\begin{aligned}
\frac{\pi \zeta(s)}{\sin \pi s}= & \sum_{k=2}^{\infty} \frac{(-1)^{k} \zeta(k)}{k(k-s)}+\frac{1-\gamma}{s-1}-\frac{1}{(s-1)^{2}}-\frac{1}{2 s^{2}}-\frac{1}{2 s} \log 2 \pi \\
& +\sum_{k=1}^{M} \frac{\zeta(1-2 k)}{1-2 k} \frac{1}{s-(1-2 k)}+\int_{1}^{\infty} H_{M}(z) z^{-s-1} d z,
\end{aligned}
$$

where

$$
H_{M}(z)=O\left(\frac{B_{M+1}}{(M+1)(2 M+1)} \quad \frac{1}{2 M+\sigma+1}\right),
$$

where $\gamma$ is Euler's constant and $B_{M+1}$ is the $M+1$ st Bernoulli number. The integral converges for $\sigma>-M$.

Proof. The proof is quite simple and involves no complexity.

We start with

$$
\frac{1}{\Gamma(z)}=z e^{\gamma z} \prod_{1}^{\infty}\left(1+\frac{z}{n}\right) e^{-z / n}
$$

If $|z|<1$, then

$$
\begin{aligned}
\log \frac{e^{-\gamma z}}{\Gamma(z+1)} & =\sum_{n=1}^{\infty}\left(\log \left(1+\frac{z}{n}\right)-\frac{z}{n}\right) \\
& =\sum_{n=1}^{\infty} \sum_{k=2}^{\infty} \frac{(-1)^{k-1} z^{k}}{k n^{k}} \\
& =\sum_{k=2}^{\infty} \frac{(-1)^{k-1} z^{k}}{k} \zeta(k) .
\end{aligned}
$$

Using the calculus of residues, it can readily be shown that the right hand side of (1) is

Received by the editors July 22, 1965.

1 The author is unable to find any similar formula in the literature.

2 Compare with the so-called Cardinal series. 


$$
\frac{1}{2 \pi i} \int_{3 / 2-i \infty}^{3 / 2+i \infty} \frac{\pi z^{8} \zeta(s)}{s \sin \pi s} d s,
$$

provided that $|\arg z| \leqq \pi-\delta, \delta>0$. Consequently

$$
\log \frac{e^{-\gamma z}}{\Gamma(1+1 / z)}=\frac{1}{2 \pi i} \int_{3 / 2-i \infty}^{3 / 2+i \infty} \frac{\pi z^{-s} \zeta(s)}{s \sin \pi s} d s,
$$

subject to the above conditions.

If $s=\sigma+i$ is not an integer and $\sigma>1$, we can apply a suitable form of the Mellin transform to get

$$
\begin{aligned}
\frac{\pi \zeta(s)}{s \sin \pi s} & =\lim _{\lambda \rightarrow \infty} \int_{1 / \lambda}^{\lambda} \log \frac{e^{-\gamma z}}{\Gamma\left(1+z^{-1}\right)} z^{8-1} d z \\
& =\lim _{\lambda \rightarrow \infty}\left(\int_{1 / \lambda}^{1}+\int_{1}^{\lambda}\right) \log \frac{e^{-\gamma z}}{\Gamma\left(z^{-1}+1\right)} z^{-1} d z \\
& =A+B .
\end{aligned}
$$

On the other hand, it is easily verified that

$$
\begin{aligned}
A & =\lim _{\lambda \rightarrow \infty} \sum_{k=2}^{\infty} \frac{(-1)^{k}}{k} \zeta(k) \int_{1 / \lambda}^{1} z^{k-s-1} d z \\
& =\sum_{k=2}^{\infty} \frac{(-1)^{k}}{k(k-s)} \zeta(k),
\end{aligned}
$$

the interchange of limiting processes presenting no difficulty. Consequently since $B$ converges for $1<\sigma<2$, we conclude that

$$
\frac{\pi \zeta(s)}{s \sin \pi s}=\sum_{k=2}^{\infty} \frac{(-1)^{k}}{k(k-s)} \zeta(k)+\int_{1}^{\infty} \log \frac{e^{-\gamma z}}{\Gamma(1+z)} z^{-s-1 !} d z
$$

The integral on the right however is

$$
\frac{-\gamma}{s-1}-\frac{1}{s^{2}}-\int_{1}^{\infty} \log \Gamma(z) z^{-8-1} d z .
$$

We apply Stirling's formula to $\log \Gamma(z)$

$$
\begin{aligned}
\log \Gamma(z)= & \left(z-\frac{1}{2}\right) \log z-z+\frac{1}{2} \log 2 \pi \\
& +\sum_{r=1}^{M} \frac{(-1)^{r-1} B_{r}}{2 r(2 r-1)} z^{-2 r+1}+H_{M}(z) \\
= & h(z)+\sum_{r=1}^{M} \frac{(-1)^{r-1} B_{r}}{2 r(2 r-1)} z^{-2 r+1}+H_{M}(z),
\end{aligned}
$$


where $h(z)=\left(z-\frac{1}{2}\right) \log z-z+\frac{1}{2} \log 2 \pi$ and

$$
H_{M}(z)=O\left(\frac{B_{M+1}}{(M+1)(2 M+1)} \frac{1}{|z|} 2 M+1\right) .
$$

$\int_{1}^{\infty} h(z) z^{-1} d z$ involves only elementary factors which can be integrated out. Having done so and combining with (6), we see that

(7)

$$
B=\frac{1-\gamma}{s-1}-\frac{1}{(s-1)^{2}}-\frac{1}{2 s^{2}}-\frac{\log 2 \pi}{2 s}
$$

$$
+\sum_{r=1}^{M} \frac{\zeta(1-2 r)}{1-2 r} \frac{1}{s-(1-2 r)}+\int_{1}^{\infty} H_{M}(z) z^{-s-1} d z .
$$

From (3), (4) and (7) we get

$$
\begin{aligned}
\frac{\pi \zeta(s)}{\sin \pi s}= & \sum_{k=2}^{\infty} \frac{(-1)^{k} \zeta(k)}{k(k-s)}+\frac{1-\gamma}{s-1}-\frac{1}{(s-1)^{2}}-\frac{1}{2 s^{2}}-\frac{\log 2 \pi}{2 s} \\
& +\sum_{k=1}^{M} \frac{\zeta(1-2 k)}{1-2 k} \frac{1}{s-(1-2 k)}+\int_{1}^{\infty} H_{M}(z) z^{-s-1} d z
\end{aligned}
$$

This formula which was shown to hold for $s$ not an integer and for $1<\sigma<2$ now holds by analytic continuation for $\sigma>-M$ in view of the estimate on $H_{M}(z)$.

Pennsylvania State University 